\title{
Archivos en 2021, segundo año de la pandemia de COVID-19
}

\author{
Archivos in 2021, second year of the COVID-19 \\ pandemic
}

\author{
Francisco Vega Álvarez \\ Jordi Delclós ${ }^{1}$ \\ Guillermo García' \\ José Miguel Martínez ${ }^{1}$ \\ José María Ramada ${ }^{1}$ \\ María del Mar Seguí \\ Elena Ronda ${ }^{\top}$
}

${ }^{1}$ Archivos de Prevención de Riesgos Laborales, Barcelona, España.

Al igual que ya sucedió en 2020, 2021 ha estado dominado por la pandemia de COVID-19. Si hace un año escribíamos esta Nota Editorial con gran preocupación por la evolución de la situación de la infección originada por el SARS-CoV-2 y, a su vez, con grandes esperanzas puestas en las vacunas como estrategia preventiva ${ }^{(1)}$, la actual viene marcada por la incertidumbre generada por la irrupción de la nueva variante de preocupación: Omicrón ${ }^{(2)}$.

Son varios los estudios que, durante estos meses, han puesto de manifiesto la importancia del lugar de trabajo, tanto como factor de riesgo para adquirir la infección, como por la relevancia de disponer y usar las medidas de protección para disminuir y controlar su expansión $n^{(3,4)}$. Una reciente editorial(5) identificaba las tres principales líneas de investigación en salud laboral derivadas de la pandemia: 1. Identificación de ocupaciones con mayor riesgo y qué condiciones laborales específicas contribuyen a los riesgos. Este objetivo se reconoce como especialmente valioso para la preparación ante amenazas de pandemias futuras. 2. El impacto que la COVID-19 ha introducido en el cómo, dónde y cuándo trabajamos, y las consecuencias para los trabajadores de la salud, afectando especialmente su salud mental. 3. El impacto de la pandemia en las desigualdades sociales. Como se puede ver en la editorial que se publica en este número que analiza de forma específica el caso de los trabajadores temporeros ${ }^{(6)}$.

Archivos, como ya anunciamos, asumió desde el primer momento el reto de servir de referencia en la transmisión de conocimiento relevante para la salud laboral(1), en esta Nota repasamos las actividades realizadas a lo largo de 2021. En primer lugar, hay que informar que Archivos se ha convertido en la revista científica de 
la Asociación Española de Higienistas Industriales (AEHI), de esta forma contribuimos a reforzar nuestro objetivo de dar respuesta a la mejora de la práctica de la prevención de riesgos laborales: la protección de la salud y la seguridad de los trabajadores desde todos sus ámbitos. En segundo lugar, nuestra revista ha sido incluida como media partner de la Agencia Europea de Seguridad y Salud en el Trabajo; esperemos que esta iniciativa que aglutina a periodistas y editores de los Estados miembros de la Unión Europea interesados en promover activamente la seguridad y la salud en el lugar de trabajo, contribuya a incrementar la visibilidad y la transferencia de los resultados de los artículos (Figura 1). En tercer lugar, anunciar que hemos entrado en dos nuevos repositorios. REDIB, plataforma de contenidos científicos y académicos en acceso abierto producidos en el ámbito iberoamericano con más de 15000 usuarios registrados y PUBLINDEX sistema diseñado por Colciencias para la evaluación y clasificación de revistas científicas en Colombia. Es una petición de investigadores latinoamericanos, ya que estas bases de datos son consideradas en los sistemas de acreditación de muchos de estos países, por lo que esperamos tenga un reflejo en la recepción de manuscritos. Por último, este año solicitamos la evaluación de la calidad editorial y científica (conocido como "sello de calidad de la FCYT"). Es un sistema que se basa en doce indicadores de obligado cumplimiento. Archivos puntuó favorablemente en once de ellos (identificación de los miembros de los consejos en la publicación, apertura del Consejo de Redacción, existencia de instrucciones detalladas a los autores, existencia de resúmenes y palabras clave, traducción de los títulos de los artículos, palabras clave y resúmenes al inglés, declaración y cumplimiento de la periodicidad, existencia de arbitraje científico externo, internacionalidad, mayoría de artículos de investigación entre los contenidos publicados, inexistencia de endogamia en las autorías y existencia de políticas de acceso abierto) pero en uno no alcanzó la puntuación positiva requerida para su alcance: nivel de repercusión, impacto y visibilidad de la revista. Esto se resume en que el número de citas de los artículos de Archivos es muy bajo, y se necesita una mayor repercusión, y, sobre todo, en artículos publicados en revistas indexadas en el Journal Citation Report. Como llevamos haciendo los diferentes equipos editoriales ${ }^{(7,8)}$, seguiremos trabajando para lograrlo, y recordando la relevancia de utilizar artículos de Archivos en las referencias bibliográficas de los trabajos publicados en otras revistas, nacionales e internacionales, siempre que sean relevantes. También vamos a abrir, a partir del nuevo año, la posibilidad de publicar artículos en español y en inglés. Será responsabilidad de la autoría que los artículos o resúmenes utilicen un inglés correcto y de calidad. 


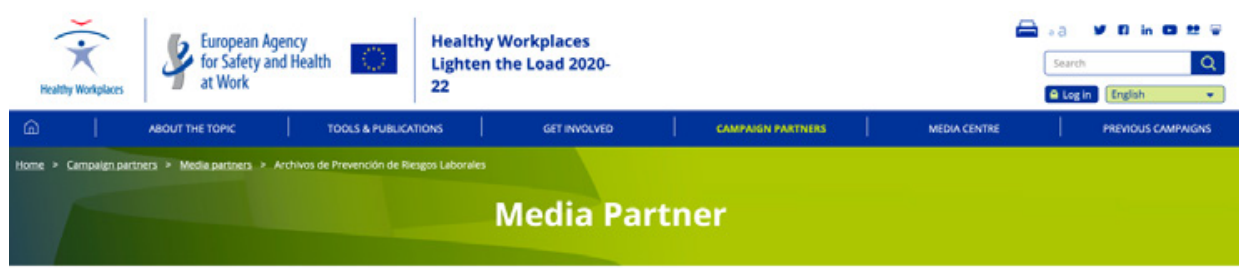
Archivos de Prevención de Riesgos Laborales

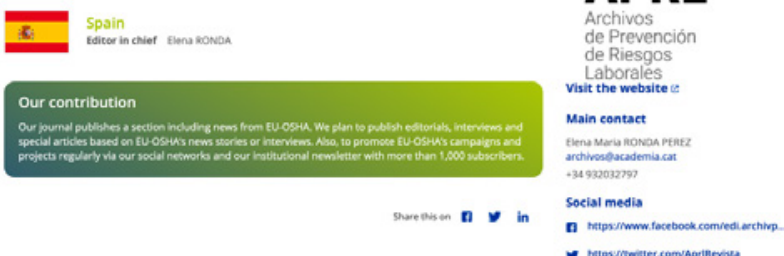

Figura 1: Web de la EU-OSHA en la que Archivos de Prevención de Riesgos Laborales figura como media partner. Fuente: https://healthy-workplaces.eu/en/campaign-partners/archivos-de-prevencion-de-riesgos-laborales?fbclid=IwAR1XR-VBj0-cZ6r3sY3F2Sowzc1VD_cjsgDzkNXvhvYUqfUSrDIdEhb2r5w

\section{Proceso editorial}

\section{Gestión de manuscritos}

Durante 2021, el número de manuscritos gestionados se ha incrementado respecto al del año anterior, aunque el número de aceptados prácticamente se mantiene (Tabla 1).

Tabla 1: Manuscritos a gestionados en Archivos de Prevención de Riesgos Laborales (2019 a 2021, volúmenes 22, 23 y 24).

\begin{tabular}{lccc}
\hline & $\mathbf{2 0 1 9}$ & $\mathbf{2 0 2 0}$ & $\mathbf{2 0 2 1}$ \\
\hline Aceptados & 22 & 25 & 26 \\
\hline Rechazados & 14 & 21 & 24 \\
\hline Abandonos & 4 & 0 & 1 \\
\hline En proceso & 6 & 12 & 10 \\
\hline Total & 46 & 58 & 61 \\
\hline
\end{tabular}

ase incluyen editoriales, originales, originales breves, artículos especiales, revisiones, artículos de opinión,

estudios de casos, notas técnicas y cartas al director hasta el 16 de diciembre de 2021.

bos manuscritos en proceso se contabilizaron en el momento de elaborarse las Notas Editoriales de los

correspondientes años.

En la Tabla 2 se presenta la distribución por tipo de trabajo publicado. Se mantiene la tendencia ascendente del número de originales publicados con respecto al año anterior. Además, cabe destacar que la suma de los trabajos que se someten a 
revisión por pares (originales, revisiones y estudios de casos) supera el 50\% de los contenidos publicados.

Tabla 2: Tipos de artículos a publicados en Archivos de Prevención de Riesgos Laborales (2019 a 2021).

\begin{tabular}{|c|c|c|c|}
\hline & 2019 & 2020 & 2021 \\
\hline Editoriales & 4 & 6 & 5 \\
\hline Notas editoriales & 1 & 1 & 1 \\
\hline Originales ${ }^{b}$ & 7 & 11 & 12 \\
\hline Revisiones & 0 & 3 & 2 \\
\hline Estudios de casos & 2 & 2 & 1 \\
\hline Notas Técnicas & 2 & 0 & 0 \\
\hline Cartas a la directora & 0 & 2 & 3 \\
\hline Otros $^{\circ}$ & 4 & 3 & 4 \\
\hline
\end{tabular}

Excluye secciones informativas (Sociedades, Noticias, Archivos Selección, Archivos Evidencia, Archivos

Formación)

bIncluye Originales Breves.

○tros: Comunicaciones de la Diada de la @SCSL, Artículos Especiales, Artículos de Opinión, eto

En cuanto a los tiempos de gestión del proceso editorial (Figura 2), las medianas de los tres indicadores (recepción-aceptación, aceptación-publicación, y recepción-publicación) se han reducido respecto al año anterior. Este descenso, probablemente, se corresponde con un alivio de la sobrecarga laboral provocada en 2020 por la situación devenida por la pandemia que afectó tanto a autores como a evaluadores y editores. Sin embargo, algunos manuscritos concretos se han visto afectados por retrasos específicos y podría explicar el ascenso observado en la mediana de la recepción a la publicación que este año alcanzó los 197 días. De todas formas, trabajaremos en acortar los tiempos de gestión como objetivo para este nuevo año.
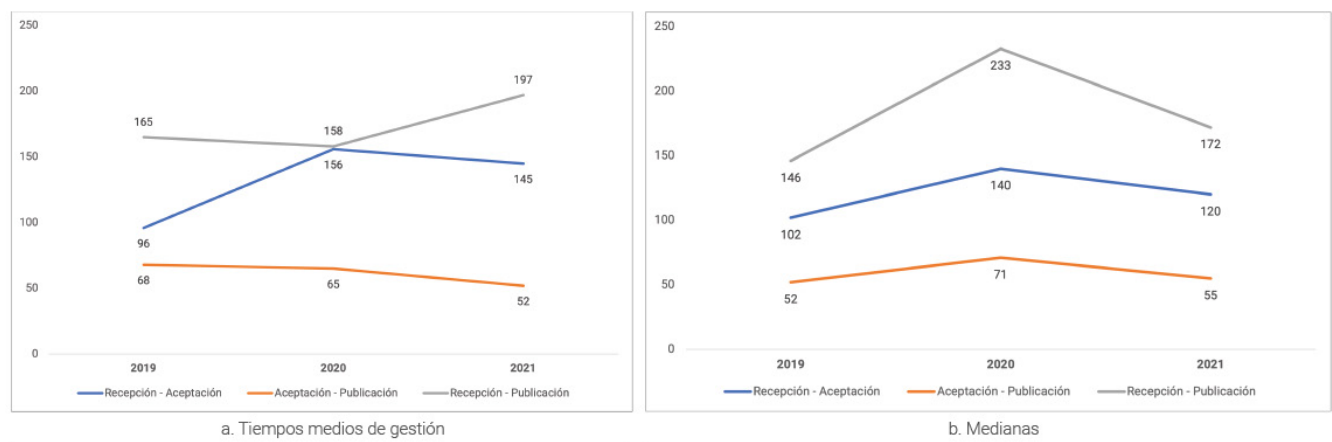

Figura 2: Evolución de los tiempos medios de gestión y medianas (días) de los manuscritos recibidos en Archivos de Prevención de Riesgos Laborales (2019 a 2021). Incluye solo originales, originales breves, revisiones y estudios de casos. 


\section{Autorías y contenidos}

Durante el 2021, han escrito en la revista casi la misma proporción de autoras (48\%) y autores (52\%), y la procedencia geográfica ha sido mayoritariamente española, destacamos la participación desde 4 países latinoamericanos (Tabla 3). En cuanto a autorías nacionales, Cataluña es la comunidad con más presencia, seguida de la Comunidad Valenciana y el País Vasco.

Tabla 3: Características de los autores que han publicado en APRL durante el año 2021. Excluye secciones informativas (Sociedades, Noticias, Archivos Selección, Archivos Evidencia, Archivos Formación).

\begin{tabular}{|c|c|c|}
\hline & $\mathbf{n}$ & $\%$ \\
\hline \multicolumn{3}{|l|}{ Género } \\
\hline Hombres & 43 & 51,81 \\
\hline Mujeres & 40 & 48,19 \\
\hline \multicolumn{3}{|l|}{ País de procedencia } \\
\hline España & 63 & 75,90 \\
\hline Ecuador & 2 & 2,41 \\
\hline Bélgica & 1 & 1,20 \\
\hline Colombia & 2 & 2,41 \\
\hline Perú & 10 & 12,05 \\
\hline Reino Unido & 1 & 1,20 \\
\hline Chile & 4 & 4,82 \\
\hline \multicolumn{3}{|c|}{ Comunidad Autónoma de procedencia (autores españoles) } \\
\hline Andalucía & 1 & 1,59 \\
\hline Castilla-La Mancha & 1 & 1,59 \\
\hline Cataluña & 31 & 49,21 \\
\hline Comunidad Valenciana & 11 & 17,46 \\
\hline Islas Canarias & 3 & 4,76 \\
\hline Madrid & 3 & 4,76 \\
\hline Navarra & 3 & 4,76 \\
\hline País Vasco & 10 & 15,87 \\
\hline \multicolumn{3}{|l|}{ Instituciones } \\
\hline Centros de investigación (incluye universidades) & 43 & 47,25 \\
\hline Servicios de Prevención & 2 & 2,20 \\
\hline Administración sanitaria & 10 & 10,99 \\
\hline Administración laboral & 7 & 7,69 \\
\hline Organizaciones sindicales & 0 & 0,00 \\
\hline Centros sanitarios & 20 & 21,98 \\
\hline Sociedades científicas & 9 & 9,89 \\
\hline
\end{tabular}


Un año más, la revista sigue manteniendo su vocación transversal (Tabla 3), lo que se manifiesta en la presencia notable de autoras y autores que trabajan en centros sanitarios, miembros de sociedades científicas o pertenecen a la administración. Y continuando con la dinámica de los últimos años, prácticamente la mitad de los autores están vinculados a universidades o centros de investigación.

Los contenidos más frecuentemente abordados (Figura 3), como viene siendo habitual, son los relativos a políticas y estrategias para la prevención y problemas de salud relacionados con el trabajo.

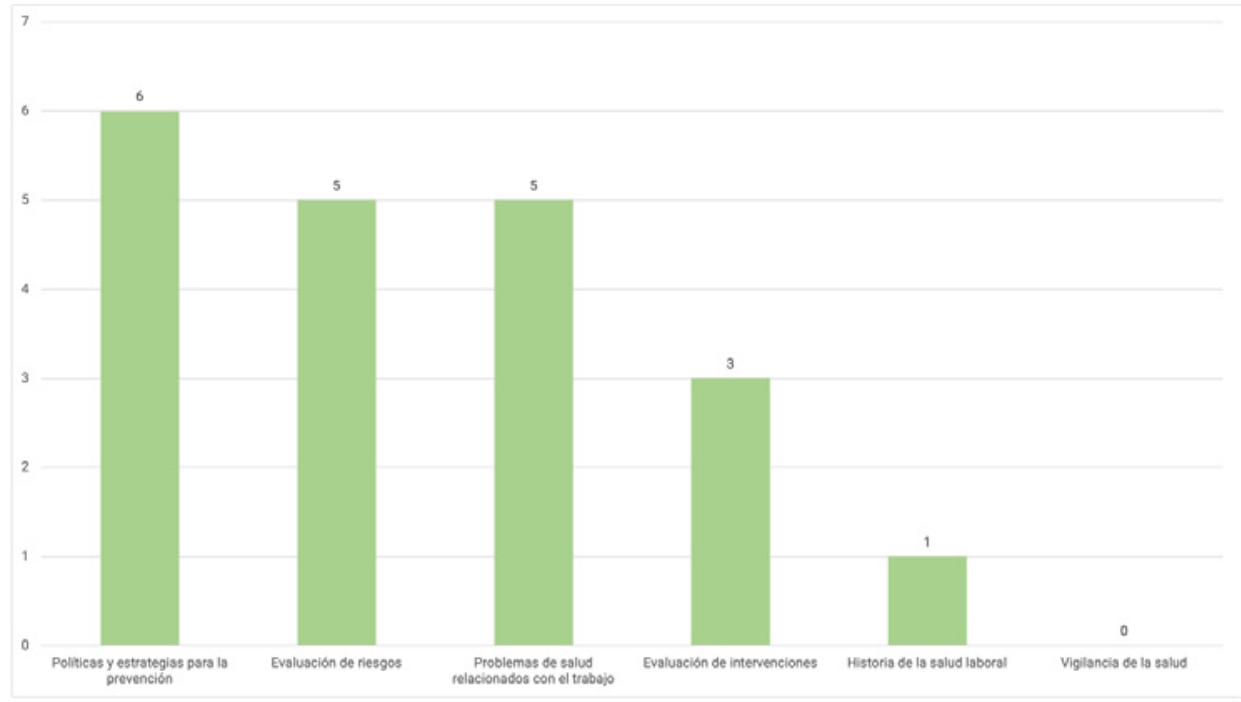

Figura 3: Distribución de los artículos publicados en APRL durante el año 2021 según el tipo de contenido. Excluye secciones fijas (Noticias, Archivos Selección, Archivos Evidencia, Archivos Formación), notas editoriales y comunicaciones de la Diada de la @SCSL).

\section{Tráfico web y presencia en redes}

Open Journal Systems (OJS), el sistema de gestión web adoptado por la revista hace dos años ${ }^{(9)}$, permite obtener varios tipos de estadísticas sobre el tráfico web.

La Figura 4 muestra la evolución de consultas a los resúmenes de los trabajos desde el 1 de enero de 2020, fecha en la que se comienza a utilizar OJS. En ella, se aprecia que la media de consultas mensuales es de aproximadamente 2000, superando los 4000 en abril de 2020 y octubre 2021. 


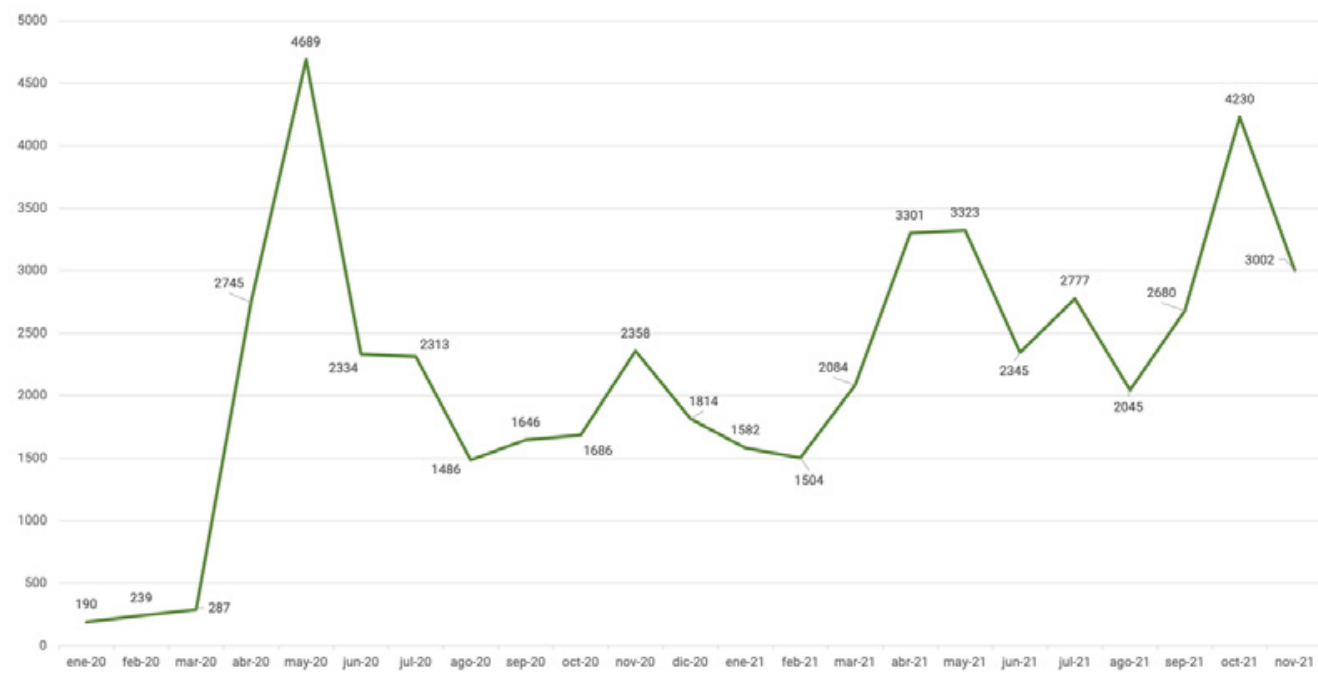

Figura 4: Evolución de la cifra mensual de consultas a los resúmenes de los trabajos publicados (enero 2020-noviembre de 2021).

A través de un código de seguimiento de Google Analytics, se permite obtener información ampliada sobre los visitantes del sitio web: por ejemplo, su ubicación, género, edad o idioma. La figura 5 muestran que durante 2021 la web de Archivos ha sido visitada por más de 15000 personas desde 145 países. Si bien la mayor parte de las visitas se producen desde España y Latinoamérica, debe resaltarse que han consultado nuestra web casi mil personas en Estados Unidos y más de 500 en China.

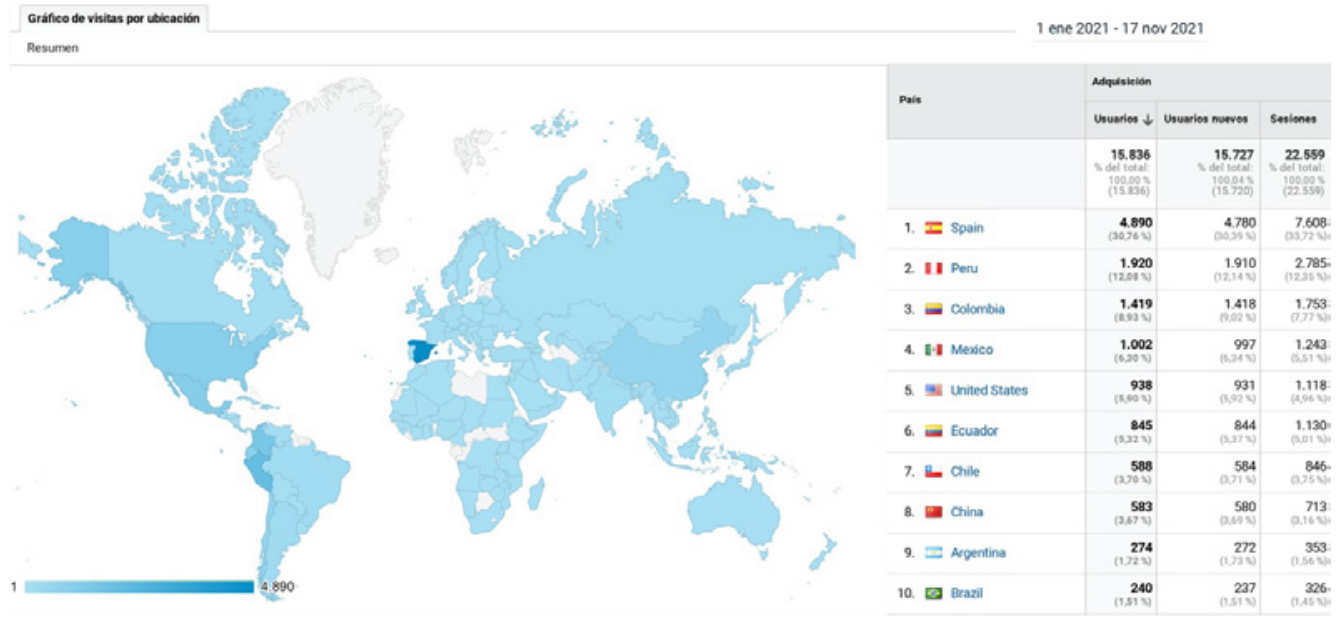

Figura 5: Informe de las visitas a la web según ubicación. Fuente: Google Analytics. 
Para ayudar a difundir los contenidos que se publican, e incrementar su visibilidad, la revista tiene cuentas en dos redes sociales Facebook (180 seguidores) y Twitter (67 seguidores). En los próximos años, pondremos en marcha campañas específicas con las que esperamos incrementar nuestra presencia en dichas redes.

Queremos finalizar estas líneas expresando nuestro agradecimiento a todas las personas que intervienen para que cada número de la revista salga a la luz y a incrementar su calidad: los distintos comités, evaluadores externos - que son reconocidos públicamente en Anexo I de esta Nota-, y a los coordinadores de Archivos Evidencia y Archivos Selección -Consol Serra, Mar Seguí y Guillermo García- y de la sección de noticias, Amàlia Valls. Queremos también mencionar, en especial, a Laura Fontcuberta, que hasta este 2021 y a lo largo de estos años fue la editora técnica de Archivos. Muchas gracias por su gran labor, por todo el trabajo realizado y por su dedicación. Un agradecimiento especial también a Motu Estudio por su disponibilidad y ayuda en la gestión de la plataforma y en la publicación de cada número. El apoyo de la Junta de la Associació Catalana de Salut Laboral (@SCSL) así como de sus socios es inestimable para la existencia de Archivos. Y, claro está, a nuestros autores y lectores, el verdadero estímulo para seguir con la tarea del día a día. A todos ellos, les invitamos a asomarse por nuestra web, así como a seguirnos en Facebook y Twitter.

Recibid nuestros mejores deseos para el próximo año.

\section{Bibliografía}

1. Fontcuberta L, Delclós J, Guillermo G, Ramada JM, Seguí M, Ronda E. 2020: Archivos en tiempos de la COVID-19. Arch Prev Riesgos Labor. 2021;24(1):12-9. doi: 10.12961/aprl.2021.24.01.02.

2. Karim SSA, Karim QA. Omicron SARS-CoV-2 variant: a new chapter in the COVID-19 pandemic. Lancet. 2021;398(10317):2126-8. doi: 10.1016/S01406736(21)02758-6.

3. Hawkins D. Differential occupational risk for COVID-19 and other infection exposure according to race and ethnicity. Am J Ind Med. 2020;63(9):817-20. doi: 10.1002/ajim.23145.

4. Zhang M. Estimation of differential occupational risk of COVID-19 by comparing risk factors with case data by occupational group. Am J Ind Med. 2021;64(1):3947. doi: 10.1002/ajim.23199.

5. Burdorf A, Porru F, Rugulies R. The COVID-19 (Coronavirus) pandemic: consequences for occupational health. Scand J Work Environ Health. 2020;(3):229-30. 10.5271/sjweh.3893.

6. Briones-Vozmediano E, González-González A. Explotación y precariedad sociolaboral, la realidad de las personas migrantes trabajadoras en agricultura en España. Arch Prev Riesgos Labor. 2022;25(1):18-24 doi: 10.12961/aprl.2022.25.01.01. 
7. Benavides FG, García AM, Ronda E. 20 años de investigación en salud laboral,... sostiene Archivos (1998-2018). Arch Prev Riesgos Labor. 2018;21(2):78-9. doi: 10.12961/aprl.2018.21.02.2.

8. Ronda E, García AM, Schlaghecke JC. Archivos, mucho ya hecho y mucho por hacer. Arch Prev Riesgos Labor. 2015;18(4):183-4. doi: 10.12961/aprl.2015.18.4.02.

9. Fontcuberta L, Delclós J, García G, Martínez JM, Ramada JM, Ronda E. Archivos 2019: Bienvenido OJS, apuesta decidida por la calidad y la visibilidad editorial. Arch Prev Riesgos Labor. 2020;23(1):14-21. doi: 10.12961/aprl.2020.23.01.02.

\section{Anexo I: Evaluadores externos para Archivos de Prevención de Riesgos Laborales (2021)}

\begin{tabular}{|c|c|c|}
\hline Nombre & Apellidos & Afiliación institucional \\
\hline Mar & Blasco & Universidad de Alicante, Alicante, España \\
\hline Joan & Ingles Torruella & $\begin{array}{l}\text { Associació Catalana de Salut Laboral, Barcelona, } \\
\text { España }\end{array}$ \\
\hline Julio & Lavarello-Salinas & Universitat de Valencia, Valencia, España \\
\hline Mireia & Utzet Sadurni & $\begin{array}{l}\text { Centre d'Investigació en Salut Laboral, } \\
\text { Universidad Pompeu Fabra, Barcelona, España }\end{array}$ \\
\hline Claudia & Palma-Vasquez & $\begin{array}{l}\text { Centre d'Investigació en Salut Laboral, } \\
\text { Universidad Pompeu Fabra, Barcelona, España }\end{array}$ \\
\hline Amaia & Ayala Garcia & $\begin{array}{l}\text { Centre d'Investigació en Salut Laboral, } \\
\text { Universidad Pompeu Fabra, Barcelona, España }\end{array}$ \\
\hline Julià & Del Prado Vinuesa & $\begin{array}{l}\text { Centre d'Investigació en Salut Laboral, } \\
\text { Universidad Pompeu Fabra, Barcelona, España }\end{array}$ \\
\hline Diego Jose & Lopez Rodriguez & Master en PRL, España \\
\hline Beatriz & Calvo-Cerrada & Universidad Pompeu Fabra, Barcelona, España \\
\hline Rosa Maria & Orriols Ramos & $\begin{array}{l}\text { Tècnica superior en PRL. Licenciada en CC } \\
\text { Químicas, España }\end{array}$ \\
\hline Francisco & Brocal & Universidad de Alicante, Alicante, España \\
\hline Erica & Briones Vozmediano & Universidad de Lleida, Lleida, España \\
\hline José María & Ramada Rodilla & Hospital Parc de Salut Mar, Barcelona, España \\
\hline Helena & Thomas Currás & Universidad Rey Juan Carlos, Madrid, España \\
\hline Fermín & Torrano Montalvo & $\begin{array}{l}\text { Universidad Internacional de La Rioja, Logroño, } \\
\text { España }\end{array}$ \\
\hline Miguel Angel & Burgos Flores & Instituto Nacional de Salud, Lima, Perú \\
\hline Carlos & Montes & $\begin{array}{l}\text { Escuela Universitaria de Relaciones Laborales. } \\
\text { Universidad de Santiago de Compostela, } \\
\text { Santiago de Compostela, España }\end{array}$ \\
\hline Javier & González Caballero & $\begin{array}{l}\text { Instituto Nacional de la Seguridad Social. Servicio } \\
\text { de Prevención y Salud Laboral, Bilbao, España }\end{array}$ \\
\hline Paula & Benavidez Lozano & Universidad de Alicante, Alicante, España \\
\hline
\end{tabular}




\begin{tabular}{|c|c|c|}
\hline Nombre & Apellidos & Afiliación institucional \\
\hline Ángela & Sanjuan & Universidad de Alicante, Alicante, España \\
\hline Rosa & Esteve & Universidad de Alicante, Alicante, España \\
\hline $\begin{array}{l}\text { Meritxell } \\
\text { Montserrat }\end{array}$ & Corral Luque & $\begin{array}{l}\text { Servicio de Salud Laboral, Parc de Salut Mar, } \\
\text { Barcelona, España }\end{array}$ \\
\hline Pablo & Gutierrez & Universidad Tecnológica del Perú, Lima, Perú \\
\hline Pilar & Díaz Pérez & $\begin{array}{l}\text { Servicio de Salud Laboral, Parc de Salut Mar, } \\
\text { Barcelona, España }\end{array}$ \\
\hline Alfonso & Apellaniz González & $\begin{array}{l}\text { Facultad de Medicina y Odontología, Universidad } \\
\text { del País Vasco, España }\end{array}$ \\
\hline Susana & Ruiz Vicente & Parc de Salut Mar, Barcelona, España \\
\hline Cristina & Alvarado Oviedo & $\begin{array}{l}\text { Hospital del Mar, Parc de Salut MAR, Barcelona, } \\
\text { España }\end{array}$ \\
\hline Ana & Beltran Fonollosa & $\begin{array}{l}\text { Ferrocarrils de la Generalitat de Catalunya, } \\
\text { Barcelona, España }\end{array}$ \\
\hline Leyre & De La Peña Perea & $\begin{array}{l}\text { Associació Catalana de Salut Laboral, Barcelona, } \\
\text { España }\end{array}$ \\
\hline Eva & González Menéndez & $\begin{array}{l}\text { Universidad Internacional de La Rioja, Logroño, } \\
\text { España }\end{array}$ \\
\hline Vega & García López & $\begin{array}{l}\text { Instituto de Salud Publica y Laboral de Navarra, } \\
\text { Pamplona, España }\end{array}$ \\
\hline Susana & Ruiz Vicente & Parc de Salut Mar, Barcelona, España \\
\hline Alba & De Juan Pérez & Universidad de Alicante, Alicante, España \\
\hline Encarnación & Martínez Fernández & $\begin{array}{l}\text { Consorci Sanitari Integral (CSI), L'Hospitalet de } \\
\text { Llobregat, Barcelona, España }\end{array}$ \\
\hline Teresa & Gea & $\begin{array}{l}\text { Servicio Medicina Preventiva Hospital de San } \\
\text { Juan de Alicante, Alicante, España }\end{array}$ \\
\hline Ikram & Benazizi & Universidad Miguel Hernández, Elche, España \\
\hline Victoria & Roca & Universidad de Alicante, Alicante, España \\
\hline Tatiana & Cuervo & $\begin{array}{l}\text { Universidad Internacional de La Rioja, Logroño, } \\
\text { España }\end{array}$ \\
\hline Natalia & Orviz Martínez & $\begin{array}{l}\text { Universidad Internacional de La Rioja, Logroño, } \\
\text { España }\end{array}$ \\
\hline Adriana & Oliva & Universidad de Alicante, Alicante, España \\
\hline Ángela & Sanjuan & Universidad de Alicante, Alicante, España \\
\hline Luis Manuel & Blanco Donoso & $\begin{array}{l}\text { Facultad de Psicología, Universidad Autónoma de } \\
\text { Madrid, Madrid, España }\end{array}$ \\
\hline
\end{tabular}

\section{¡Con nuestro reconocimiento y gratitud!}

